\title{
E-botanist system for agricultural applications
}

\begin{abstract}
Many of the existing plant species on the earth are still unknown. There are different kinds of plant species and it has been subject of interest on how to identify their species. However, the current practice to identify and distinguish each species is heavily dependent on botanists. It is inefficient and wasteful of resources in terms of time and money. Moreover, our human sensory system's limitation such as physical, mental health, fatigue and other body conditions will highly influenced the classification and identification accuracy. The ability to classify distinctive odor pattern for aromatic plants species especially for herbs provides significant impact in food industry. Each herbs species has a unique physicochemical and a distinctive odors. However, it is difficult for botanists to recognize herbs based on aroma for the species under the same family. Thereupon, the herbs odors under the same family which is the physical appearance may look almost the same characteristic and also may be having the almost same aromas. E-botanist instruments, derived from numerous types of aroma sensor technologies have been developed for a diverse of applications in a broad field of agriculture including for herbs. The intervention of electronic botanist (E-botanist) system was capable to reproduced human senses using sensor arrays and pattern recognition systems.
\end{abstract}

Keyword: E-botanist; E-nose; E-tongue 